\title{
Natur als Gesundheitsressource
}

\author{
Die Natur beeinflusst die menschliche Gesundheit in hohem Masse positiv. \\ Wieviel oder wie wenig natürliche Ressourcen braucht der Mensch? Mehrere \\ interdisziplinäre Arbeitsinitiativen zeigen aktuell, dass diese philosophisch \\ anmutende Frage kein Rätsel bleiben muss, sondern von verschiedenen \\ Seiten her ausgeleuchtet wird. Das neue Arbeitsfeld nennt sich «Green Care» \\ und expandiert rasant.
}

Yvonne Christ, Cornel Wick*

* Zentralvorstandsmitglied der Ärztinnen und Ärzte für Umweltschutz
Korrespondenz:

Yvonne Christ, Dipl. Ing. FH

Hochschule Wädenswil

Grüental

Postfach

CH-8820 Wädenswil

y.christ@hsw.ch
Der diesjährige sehr heisse Frühling ist schon in weiter Ferne, und der Sommer geht ins Land. Die Kornfelder reifen, die Menschen flanieren in den Strassen und Pärken, und wem nicht das Ozon zu schaffen macht, der erfreut sich am warmen Sonnenschein und dem Vogelgezwitscher. Die Seeufer und Flüsse werden häufig aufgesucht und laden zum Bade. Berge werden erklommen, und Bauern bestellen ihre Äcker für die Nahrungsmittel- und Tierfutterproduktion. Touristen reisen durch abwechslungsreiche Landschaften; der Herbst kommt mit grossen Schritten, und das Leben scheint in Ordnung zu sein ...

Nur wenige Menschen nehmen die Einzelheiten der Ressource «Natur» bewusst wahr. Unterschiedliche Faktoren wie Klima, geologische Vorkommen und eine kulturell geprägte Bewirtschaftung begründen das Aussehen unserer Grünflächen, sei es nun Ackerland, der Park eines Spitals oder das private Gartenbeet des Herrn Dr. Vogelfrei. Ökologische Vielfalt misst sich mit Monotonie, im kleinen wie im grossen, der Wunsch nach Rendite und Bequemlichkeit liess in den letzten Jahrzehnten Beton spriessen. Platz für neue Technologien, die dadurch verbundene Maximierung der funktionellen Effizienz sowie neue Hygienemassnahmen führten im 20. Jahrhundert zu einem Verlust an Beachtung der Raumqualität [1].

Wieviel oder wie wenig natürliche Ressourcen braucht der Mensch? Die bislang eher philosophisch anmutende Frage wird seit einiger Zeit auch wissenschaftlich untersucht. Unterschiedlichste Berufsgruppen üben sich im Dialog über so etwas Selbstverständliches wie «Natur». Kein leichtes Stück, denn eine Fülle an möglichen Herangehensweisen ergibt sich durch die Verschiedenartigkeit der persönlichen und institutionellen Vorstellungen von Natur und natürlicher Umwelt.

\section{La nature comme}

\section{ressource de santé}

La nature influence la santé humaine très positivement. Mais: combien des ressources naturelles est-ce que I'homme nécessite-il? Cette question plutôt philosophique ne doit pas rester un mystère, car plusieurs actuels travaux interdisciplinaires prouvent cette théorie. La nouvelle zone de travail s'appelle «Green Care» et développe de manière foudroyante, aussi en Suisse.

In vielen traditionellen Denkweisen bildet die Natur seit je integrativen Bestandteil ärztlichen Handelns und somit Teil von Diagnostik und Therapie. So zum Beispiel das Feng-Shui in der chinesischen Medizin, das die Interaktion der Energieflüsse zwischen Mensch und Natur beschreibt. Aber auch hierzulande gab es Völker, beispielsweise die Ägypter, die Kelten oder die alten Germanen, die über die Beeinflussung der Gesundheit an bestimmten Orten und Plätzen im Bilde waren [2].

Ende des letzten Jahrhunderts leistete Roger Ulrich, ein Landschaftsarchitekt aus Amerika, Pionierarbeit auf diesem Gebiet. Er beschäftigt sich seit Anfang der 80er Jahre mit der Frage, warum viele Leute intuitiv das Gefühl haben, der Aufenthalt in der Natur würde ihnen guttun. Etliche Studien und Reviewarbeiten sind seither erschienen. Dabei konnte eindeutig bestätigt werden, dass der blosse Anblick einer Naturszene - nur schon auf Papier - die Herzen nicht schneller schlagen lässt, sondern den Blutdruck, die Herzfrequenz und die Muskelspannung innerhalb weniger Minuten zu senken 


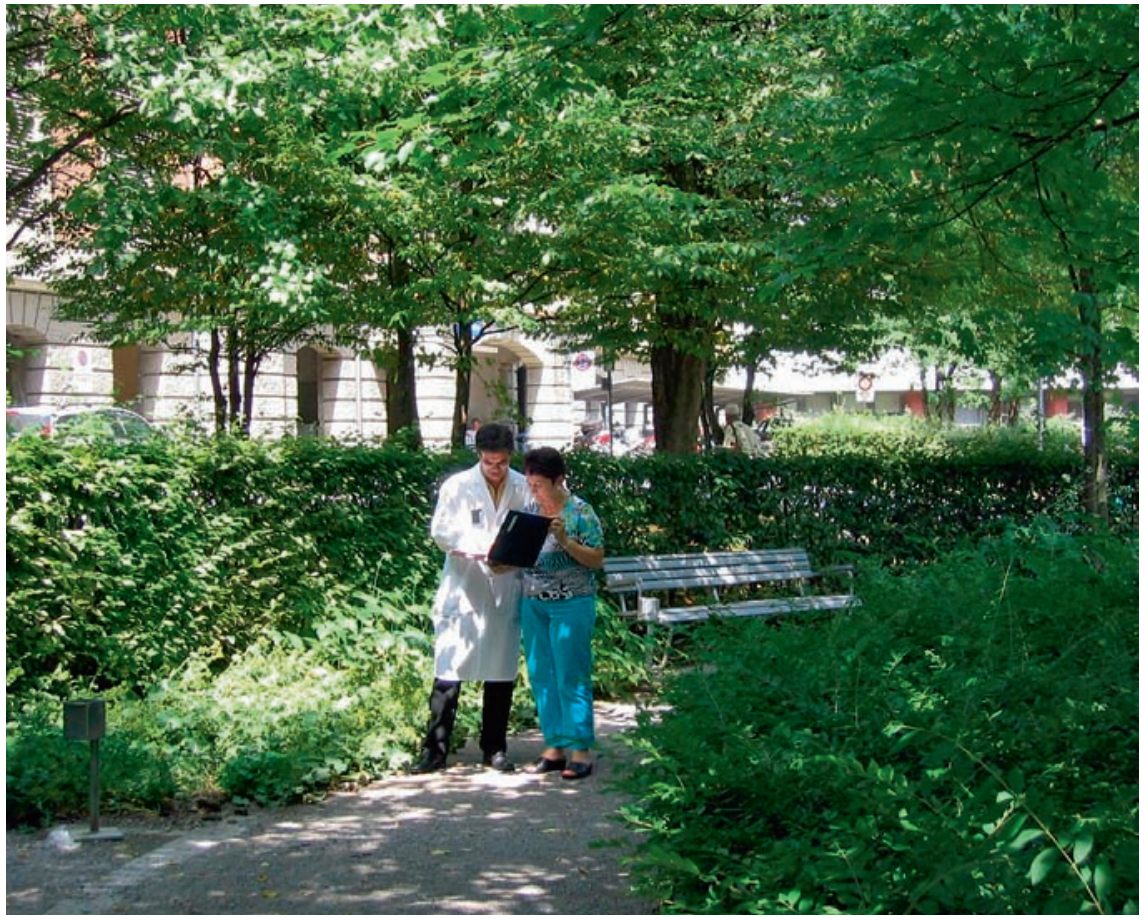

vermag [3]. Die Sicht aus dem Krankenbett beeinflusst den Heilungsverlauf ebenfalls: Ein Blick auf Grünflächen kann die Hospitalisationsdauer, den Analgetikaverbrauch und sogar die postoperativen Komplikationen vermindern [4].

Aber nicht nur das Visuelle verleiht ein positives Wohlbefinden. Die Begegnung mit Menschen (sozial), das Zirpen der Grillen (akustisch), das Blumenpflücken (taktil) oder der Duft einer schönen Blume (olfaktorisch) unterstützen diesen positiven Effekt zusätzlich [5].

Auch der gezielte, aktive Nutzen von Grünflächen wurde erkannt. Im Rahmen des Europäischen COST-Forschungsverbundes, der besonders multi- und interdisziplinäre Bereiche unterstützt, etabliert sich in der Schweiz der Begriff «Green Care» für gesundheitsfördernde, präventive und therapeutische Nutzungen der natürlichen Umwelt. Ein Zusammenschluss des Forschungsinstituts für biologischen Landbau, der Pädagogischen Hochschule Zürich und der Hochschule Wädenswil (Umwelt und natürliche Ressourcen) erarbeitet Grundlagen für spätere Anwendungen. Geplant sind Fragestellungen zu Gärten für Demenzkranke und makroökonomische Studien über die Auswirkungen von Patientenplazierungen als alternatives Standbein für landwirtschaftliche Betriebe sowie weitere Forschungsprojekte.

Schon erfolgreich durchgeführt wurde ein interdisziplinär angelegtes Projekt der Hochschulen Wädenswil, Rapperswil (Institut für Geschichte und Theorie der Landschaftsarchi- tektur) und Zürich (Soziale Arbeit). Im Alterszentrum Gibeleich in Glattbrugg ZH konnte gezeigt werden, dass die Erhaltung und Erhöhung von Lebensqualität für die Betagten über eine Neugestaltung der Aussenanlagen möglich und sinnvoll ist. Die Planung des Umschwungs wurde bis in Details der Pflanzenwahl partizipativ durchgeführt und mit dem Fachwissen der Projektpartner ergänzt.

Anhand der durch das Personal geförderten aktiven Nutzung des Gartens konnte ein «Leitfaden für Aktivierungstherapeutische Arbeiten im Garten» entwickelt werden. Als Folge der Arbeit in Gibeleich wurden zahlreiche neue Therapiegartenprojekte in Alterszentren ins Leben gerufen [6]

Der vom BAG und BUWAL (neu BAFU) 1997 ins Leben gerufene Aktionsplan Umwelt und Gesundheit (APUG) setzt sich zum Ziel, das Umwelt- und Gesundheitsverhalten eines breiten Publikums im Alltag zu verknüpfen und damit zu einer nachhaltigen Entwicklung beizutragen. Dies entspricht einer Verknüpfung der passiven und aktiven Aspekte von «Green Care». Obwohl der APUG während seiner Durchführungszeit einen volkswirtschaftlichen Nutzen von knapp 20 Mio. Franken (bei Auslagen von 14 Mio. Franken) gestiftet hat, muss er jetzt leider den Sparmassnahmen des Bundes weichen [7].

Um so wichtiger ist die Initiative weiterer Institutionen zum Thema: Durch die Lehrveranstaltung «Grün und Gesundheit», die als fakultativer Teil des Studiums Umweltingenieurwesen in Wädenswil besucht werden kann, entstehen in Zusammenarbeit mit Heimen und Spitälern studentische Arbeiten $\mathrm{zu}$ freiraumplanerischen Anwendungen für gesundheitsförderliche und therapeutische Zwecke.

An der Universität Bern wurde im Rahmen des Studiums in «Allgemeiner Ökologie» eine Projektarbeit in Zusammenarbeit mit dem Inselspital zum Thema «Healing Garden» durchgeführt [8]. Die Umgestaltung und damit die verbesserte aktive und passive Nutzung der Inselgärten soll noch dieses Jahr beginnen.

Dass die Natur die Gesundheit in hohem Masse positiv beeinflusst, wurde durch die Ende Mai 2007 präsentierte Literaturrecherche des Instituts für Sozial- und Präventivmedizin der Universität Bern untermauert [9]. Diese Studie wurde im Rahmen des neuen Projekts «Paysage à votre santé» der Stiftung Landschaftsschutz Schweiz und der Ärztinnen und Ärzten für Umweltschutz durchgeführt. In diesem Projekt werden nun Kriterien für eine möglichst gesunde Landschafts- und Siedlungsqualität erstellt. 
Leider wird diesen Effekten noch zuwenig Beachtung geschenkt. Wir hoffen natürlich, dass die neue Bewegung «Green Care» auch in der Schweiz sicheren Tritt fasst. Im Zuge der Umstrukturierungen der Fachhochschullandschaft des Kantons Zürich wird diesen Herbst in Wädenswil die neue Fachstelle «Green Care» geschaffen. Sie soll als Koordinationsplattform und Anlaufstelle für die Weiterentwicklung des jungen Wissenschaftszweiges dienen, damit in Zukunft eine aktive und passive Nutzung natürlicher Ressourcen noch viel gezielter eingesetzt werden kann - zum Wohle aller.

\section{Literatur}

1 Horsburgh CR. Healing by design. N Engl J Med. 1995;333(11):735-40.

2 Unschuld PU. Was ist Medizin? Westliche und östliche Wege der Heilkunst. München: C. H. Beck; 2003.

3 Ulrich RS. Health Benefits of Gardens in Hospitals. Paper for conference, Plants for People. International Exhibition Floriade; 2002.

Onlinepublikation: www.planterra.com/ SymposiumUlrich.pdf.
4 Ulrich RS. View through a window may influence recovery from surgery. Science. 1984;27(4647): 420-1.

5 Cooper-Marcus C, Barnes M. Healing Gardens: Therapeutic Benefits and Design Recommendations. New York: John Wiley; 1999.

6 Beck T, Schneiter-Ulmann R. Therapeutischer Garten. Ein interdisziplinäres Projekt der angewandten Forschung. Zoll+ Nr. 5. Textedition österreichischer Landschaftsplanung und Landschaftsökologie. Wien: Verlagspostamt; 2004.

7 Interface. Volkswirtschaftlicher Nutzen der Arbeit der Sektion Gesundheit und Umwelt im Bundesamt für Gesundheit (BAG). Kurzbericht zu den Ergebnissen. Luzern: Interface - Institut für Politikstudien; 2005. www.apug.ch/files/Vw_Nutzen APUG.pdf.

8 Gabriel E, Martignoli S, Röthlisberger S, Steffen M, Wick C, Wyttenbach S. Die Umgestaltung der Inselgärten nach dem Konzept Healing Garden. Bern: Universität Bern; 2005. www.ikaoe.unibe.ch/ publikationen/SR_Studentische_Arbeiten_40.pdf.

9 Abraham A, Sommerhalder K, Bolliger-Salzmann $\mathrm{H}_{\text {, }}$ Abel T. Landschaft und Gesundheit. Das Potential einer Verbindung zweier Konzepte. Bern: Universität Bern, Institut für Sozial- und Präventivmedizin; 2007. www.ispm.ch/fileadmin/doc download/ Landschaft_Gesundheit.pdf. 